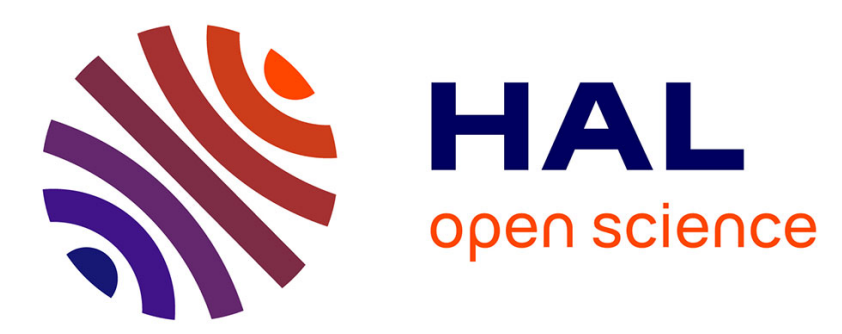

\title{
The Ionosphere Prediction Service For GNSS Users
}

Filippo Rodriguez, Roberto Ronchini, Stefano Di Rollo, Eric Guyader, Angela

Aragon-Angel, Giorgiana de Franceschi, Claudio Cesaroni, Luca Spogli, Vincenzo Romano, Marcio Aquino, et al.

\section{- To cite this version:}

Filippo Rodriguez, Roberto Ronchini, Stefano Di Rollo, Eric Guyader, Angela Aragon-Angel, et al.. The Ionosphere Prediction Service For GNSS Users. ITSNT 2018, International Technical Symposium on Navigation and Timing, Oct 2018, Toulouse, France. 10.31701/itsnt2018.25 . hal-01942268

\section{HAL Id: hal-01942268 \\ https://hal-enac.archives-ouvertes.fr/hal-01942268}

Submitted on 8 Jan 2019

HAL is a multi-disciplinary open access archive for the deposit and dissemination of scientific research documents, whether they are published or not. The documents may come from teaching and research institutions in France or abroad, or from public or private research centers.
L'archive ouverte pluridisciplinaire HAL, est destinée au dépôt et à la diffusion de documents scientifiques de niveau recherche, publiés ou non, émanant des établissements d'enseignement et de recherche français ou étrangers, des laboratoires publics ou privés. 


\title{
The Ionosphere Prediction Service for GNSS Users
}

\author{
F. Rodriguez, R. Ronchini, S. Di Rollo, Telespazio \\ E. Guyader, European Commission \\ Angela Aragon-Angel, Joint Research Centre \\ G. De Franceschi, C. Cesaroni, L. Spogli, V. Romano, INGV \\ M. Aquino, S. V. Veettil, University of Nottingham \\ F. Berrilli, D. Del Moro, A. Cristaldi, University of Tor Vergata
}

\section{BIOGRAPHIES}

Filippo Rodriguez holds the position of Project Manager in Telespazio with more than 18 years of technical and managing experience.

$\mathrm{He}$ is managing EC and ESA projects mostly focused on GNSS evolution and currently he is the coordinator of the Ionosphere Prediction Service programme for EC and the technical coordination of the Galileo PRS navigation performance monitoring tool development for the GSOp (the Galileo Service Operator).

He has two degrees in Telecommunication Engineering and Astronautics Engineering and a PHD in Control Systems Engineering. He holds two patents in GNSS technology

Stefano Di Rollo received is MSc in Telecommunication Engineering from the University of Rome La Sapienza (2008) and a II level specializing master in advanced systems for satellite communication and navigation (2010). From 2012 he was a Navigation System expert at Telespazio serving as system engineer and technical responsible on several projects regarding Galileo KPI monitoring and evolution, ionospheric activity monitoring and development of GNSS-based solutions for several applications.

Roberto Ronchini holds a M.Sc. in Computer Science and a Ph.D. in Systems Engineering both from Sapienza University of Rome.

$\mathrm{He}$ is currently employed in Telespazio as a GNSS Research Engineer and Technical Manager, and has been working on many international research projects since 2001.

His research interests in GNSS include navigation systems integrity and failure detection, ionosphere monitoring and, more in general, GNSS systems performance modelling and forecast with focus on Civil Aviation and ATM Applications.

Angela Aragon-Angel has two degrees in Mathematics and Physics and obtained her Ph.D. in the Aerospace Science and Technology Aerospace Doctoral Program of the Technical University of Catalonia (UPC) in 2010. She is currently a scientific/technical officer at the Joint Research Centre (JRC) of the European Commission (EC),
Italy. Her working topics range from ionospheric radio occultation to ionospheric modeling for positioning and navigation within the area of GNSS data processing.
ABSTRACT
Space weather events related to solar activity can affect both ground and space-based infrastructures, potentially resulting in failures or service disruptions across the globe and causing damage to equipment and systems. Global Navigation Satellite Systems (GNSS) represent one of such infrastructures that can suffer from electromagnetic phenomena in the atmosphere, in particular due to the interaction of the RF signals with the ionosphere.
The Ionosphere Prediction Service (IPS) is a project funded by European Commission to provide a prototype platform for a monitoring and prediction service of potential ionosphere-related disturbances affecting GNSS user communities. It is designed to help these communities cope with the effects of the ionospheric activity and mitigate the impacts of these effects on the specific GNSS-based application/service.
The IPS development has been conceived of two concurrent activities: the design and implementation of the prototype service and the research activity, which represents the scientific backbone of IPS and is at the base of all the models and algorithms used for the computation of the products.
The products are the basic IPS output that translate the nowcasting or forecasting information from the whole IPS system down to the final user. They are fine-tuned to match the different needs of the communities (scientific, aviation, high accuracy, etc.) which the service is targeted to and to warn the GNSS users about possible performance degradations in the presence of anomalous solar and atmospheric phenomena. To achieve this overarching aim, four different blocks of products dealing with solar activity, ionospheric activity, GNSS receiver and system performance figures have been developed and integrated into a unique service chain.
The service is available to a set of invited users since July 2018 through a web portal and its provision with all the necessary operations will last 6 months. The prototype will be also ported to the Joint Research Centre (JRC). This phase will be useful to further test the platform, and to assess whether and how a dedicated prediction service for 
Galileo users is to be implemented as part of the service facilities of the Galileo infrastructure.

\section{INTRODUCTION}

The effect of the Earth's ionosphere represents the single largest contributor to the Global Navigation Satellite System (GNSS) error budget, and abnormal ionospheric conditions can impose serious degradation on GNSS system functionality.

The effects on a GNSS receiver can include (slow or sudden) decrease of accuracy in the position and timing computations, potential loss of integrity, complete loss of one or more satellite signals, etc. With society and economies increasingly relying on the services provided by GNSS, a more thorough analysis of the impact due to those phenomena is warranted and prevention methods must be developed. This understanding can be achieved by means of the deployment of observational networks and the development of ionospheric models, which can help limiting their disruptive effects thanks to early warning alerts.

In particular, for critical and safety-related operations relying on the good performance of GNSS, like aviation, any degradation of the navigation/timing services could potentially lead to severe consequences. In the aviation domain, ionospheric events have the potential to result in hazardously misleading information, especially in the case of approach and landing operations.

The Ionosphere Prediction Service (IPS) project is developed in the framework of the Galileo Programme. It is funded by the European Union's R\&D programme Horizon 2020. The project team is composed of Telespazio (coordinator), Nottingham Scientific Ltd, Telespazio Vega DE, The University of Nottingham, The University of Rome Tor Vergata and the National Institute of Geophysics and Volcanology (INGV).

It has the objective to deliver a prototype of a monitoring and prediction service of potential ionosphere-related disturbances affecting the GNSS user communities; the main goal is to alert the GNSS users in due time of an upcoming ionospheric event potentially harmful for GNSS and for the related operations in the given application field. In the next sections an overview of the IPS project will be provided describing its main concepts, the whole service chain with the implemented architecture and the available products.

\section{THE IPS PROTOTYPE SERVICE CONCEPT}

The mission of the IPS service is to provide each of its user communities with nowcasted and forecast indicators relevant to the GNSS applications that can be affected by unexpected ionosphere behaviour [1].

This section gives a description of the IPS service concept in terms of functions and architecture designed to support the IPS mission.

\subsection{IPS Concept}

Based on the analysis of the collected user requirements, the service concept has been designed to rely on the four following functions:

1. Observations data of the Sun activity and of the ionosphere state to derive a prediction of the GNSS performance at user level in a given geographical area. This observation function requires the connection to external sensors providing the necessary data. The solar activity that is monitored consists of:

a. Flare forecasting and research on magnetic reconnection (as trigger of flares and Coronal Mass Ejections (CME));

b. Detection of solar active regions and evaluation of the flare probability using the main outcomes of magnetic reconnection models;

c. Measurements of Solar Energetic Particle (SEP) and related research activities.

2. The morphology and the dynamics of the ionospheric plasma are also studied to develop Total Electron Content (TEC) and scintillation mapping and modelling tools on different temporal and spatial scales. Data from available GNSS networks (global and regional) are used to derive ionosphere-related products;

3. Statistical approaches and Position Velocity Time (PVT) algorithms fed with ionospheric models, augmentation models and observation data to develop nowcast, short-term and long-term forecast of GNSS systems performance on global, regional and local scale;

4. Algorithm output provided in form of scalar, chart or grid values could be ingested and managed to generate and send alerts to subscribed users when it is expected that the monitored physical parameter will get outside the user-defined range. The alerts are delivered via email and displayed on a proper page of the web portal;

Other relevant IPS functions are:

- Statistical analysis. This function allows the configuration of the calculus of statistical parameters on the basic IPS products (like moments, Probability Density Functions (PDF), CDF, etc.) to be displayed on the web portal pages through one of the admissible widgets;

- Forecast retro-validation. This is a periodic report with the output of the comparison between the past forecast analysis and the corresponding actual value computed at the same time and for the same physical quantity. This function allows assessing how good the forecast algorithms actually perform in predicting the future behaviour of the monitored quantities. 


\subsection{IPS Architecture}

As shown in Figure 8 (Appendix A), the IPS logical architecture is based on the three following layers:

1. Sensors: this layer collects all the elements used to gather raw data for service products generation. Sensors are usually external to the IPS processing facilities and remotely located with respect to the RPFs. There are many types of sensors in IPS: GNSS receivers belonging to regional or global networks, on-board satellite sensors (like coronagraph), terrestrial magnetograph, etc.;

2. Remote Processing Facilities (RPFs): this layer collects all the processing entities that are logically remote respect to the central storage and distribution unit. They run all the algorithms for the generation of the user-oriented products and interact both with the remote sensors for the collection of all the needed input data and the central storage to save the generated output, to retrieve and process data from other RPFs or to trigger one or more functionalities implemented in the central unit. The current version of IPS prototype has 4 RPFs that are distinguished according to the category of generated products;

3. Central Storage and Processing Facility (CSPF): this central facility implements all the functionalities related to the storage and distribution of the products and the interaction with service users, including the transmission of notification and warnings.

\subsection{RPF Product Generation}

As said before, the RPFs are in charge to generate the forecasting and nowcasting products for the different categories, and in the following of this section a brief description of each product category is provided.

\section{RPF1 - Solar Activity Related Products}

This RPF is dedicated to the monitoring and prediction of solar events like flares, CME and solar energetic particles (SEP) linked to CME. The input data are provided by several sensors and scientific payloads, like - among the others - GOES X, SOHO, MOTH telescope etc.

RPF2 - Ionospheric Activity Related Products

This RPF is dedicated to the ionosphere monitoring where TEC and scintillation estimation are nowcasted and forecast at regional and global level and takes as input several GNSS reference stations data (e.g. IGS) and scintillation data (e.g. ISMR, RING networks).

The main scintillation parameters $\mathrm{S} 4$ and $\sigma_{\Phi}$ are measured by proper GNSS receivers on specific locations; global products are instead provided as regular maps of ROTI (Rate of TEC Index) parameter.

RPF3 / RPF4 - GNSS User Receiver and Service Related Products

The last two RPFs focus on the performances of GNSS receivers and global service. They take as input also the ionosphere estimation provided by RPF 2 and monitor and predict the GNSS related performance at local, regional and global level.

In particular, the user receiver category is dedicated to high accuracy users, while the service related category, developed by Telespazio, provides, among the others, nowcasting and forecasting of aviation related performance figures (from Aircraft Based Augmentation System (ABAS) to Satellite Based Augmentation System (SBAS)).

Table 2 in the Appendix B provides some details about the nowcasting refresh time, the forecasting temporal horizon and the spatial extent for each category.

\section{INPUT DATA SOURCES}

IPS needs several types of input data sources to generate the nowcasting and forecasting products. These sources consist of GNSS reference stations, among which scintillation monitoring stations, terrestrial sensors (like magnetometers etc.) and scientific space mission payloads. In the current prototype version of IPS, only a subset of the input data sources is under the direct control of the consortium; the sensors that are directly maintained belong to several GNSS networks. These networks are currently run by INGV and the University of Nottingham in cooperation with some hosting institutions:

- The ISMR ionospheric network of Figure 1 controls 12 GNSS stations (10 active and 2 with historical data) equipped with dual frequency receivers (NovAtel GSV4004) or with special scintillation high rate receivers $(50 \mathrm{~Hz}$ Septentrio PolaRxS). This network covers the northern Europe (Great Britain and Scandinavian peninsula), central Italy with one station in Rome and some islands in the Mediterranean sea;

- The RING geodetic network of Figure 2 consists of about 180 GPS standard dual frequency receivers distributed over Italy. The L1 and L2 signals from GPS satellite are acquired by the RING receivers at 30s sampling rate and transmitted to two main servers located at INGV premises in Rome and Grottaminarda (Southern Italy). Receiver observations in RINEX format are available with a latency of about 15 minutes and managed by a virtual machine hosted at INGV in Rome.

The IPS also uses data acquired by public GNSS data providers like IGS, EUREF and EDAS. IPS space weather monitoring and forecasting processes also depend on terrestrial and satellite sensors like magnetographs or coronagraphs. Sensors are not directly managed by the IPS researchers, but their readings can be retrieved and processed for real-time flare detection, flare and CME forecasting and SPE detection: 


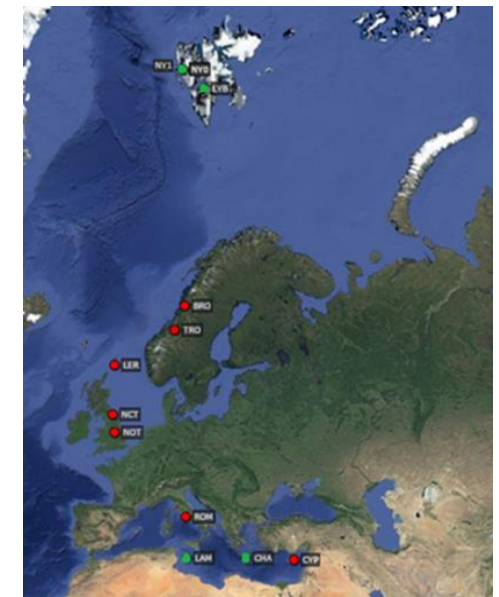

Figure 1 - ISMR Network: Receiver Locations

- NSO/GONG (Global Oscillation Network Group) H Alpha Network is a worldwide terrestrial network composed by 6 sites each equipped with a Fourier tachometer, an instrument based on a Michelson interferometer;

- MOTH (Magneto-Optical filters at Two Heights) 20 $\mathrm{cm}$ telescope equipped with $2 \mathrm{k}$ x $2 \mathrm{k}$ CMOS cameras providing magnetogram (potential field extrapolation), intensity and velocity maps using a MOF. This telescope is located at Maui Island (Hawaii);

- Helioseismic and Magnetic Imager (HMI) is an instrument designed to study oscillations and the magnetic field at the solar surface, or photosphere. HMI is one of three instruments on the Solar Dynamics Observatory (SDO) spacecraft launched on February 11, 2010;

- Atmospheric Imaging Assembly (AIA) is an instrument designed to provide an unprecedented view of the solar corona, taking images that span at least 1.3 solar diameters in multiple wavelengths nearly simultaneously, at a resolution of about 1 arcsec and at a cadence of 10 seconds or better. Together with HMI, AIA is aboard the SDO spacecraft;

- Large Angle and Spectrometric Coronagraph (LASCO) is one of a number of instruments aboard the Solar and Heliospheric Observatory satellite (SOHO).

The gathering of input raw data for the product generation, especially from public sources, is not always a smooth process, due to the unavailability of the data or the low quality of the content (errors in the bit stream, truncated data etc.). This is the most important cause of potential degradation of the IPS service. More in general, the variety of these inputs has a great impact on the design of the system interfaces, and the quality level of their providers influences the final performances of the service in terms of continuity, availability, latency, etc.

The gathering of input raw data for the product generation, especially from public sources, is not always a smooth process, due to the unavailability of the data or the low quality of the content (errors in the bit stream, truncated data etc.). This is the most important cause of potential degradation of the IPS service. More in general, the variety of these inputs has a great impact on the design of the system interfaces, and the quality level of their providers influences the final performances of the service in terms of continuity, availability, latency, etc.

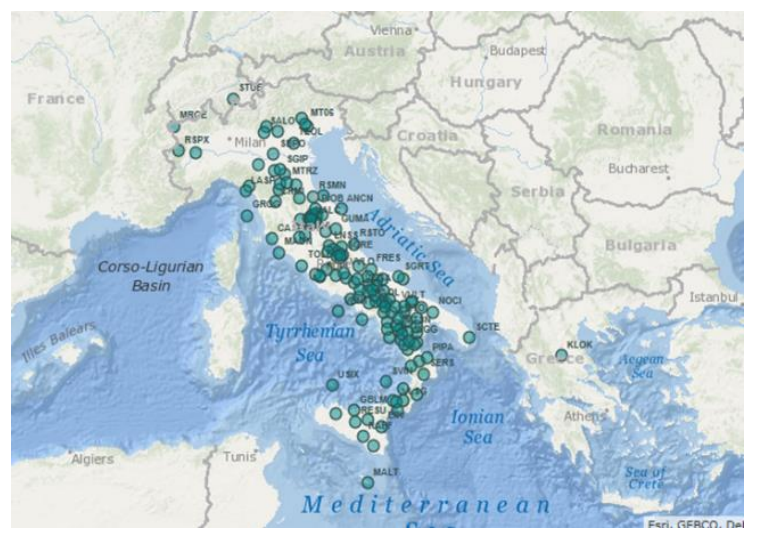

Figure 2 - RING Network: Receiver Locations

In the IPS prototype in order to cope with possible degradations of the input data, a quality control is performed in most of the algorithms, when the input data is processed via machine learning or interpolation tools to generate the final product. In this case when the number of input sources is very high, it is possible to mitigate the lack of information excluding the low quality sources relying on the redundancy of the input data. When the processing directly depends on the input data (like in the case of scintillation data or solar images), if the source has a problem this directly reflects on the output product without any possible mitigation. In this case, the related product cannot be generated during the whole time of unavailability of the input data source.

\section{THE IPS WEB PORTAL}

The IPS web portal is one of the most distinguishing components of the system and also represents the principal interface between the users and the service itself.

It has been designed to give an immediate access to the several IPS generated products to every user, allowing a high level of interaction and customization.

However, the most valuable IPS service options are available only to the registered users. The registration to IPS is free and can be requested by filling the registration form available on the project website: https: Ilwww.ips.telespazio.com.

The portal provides the user with a set of specific user community pages (Solar Physics, Ionosphere, Aviation ABAS and SBAS PA (Precision Approach), High Accuracy), offering a selection of the most interesting products for a synthetic view of the possible impacts of the solar and terrestrial atmosphere status on GNSS applications. The service administrator makes available to each user the report pages that seem to be more appropriate to its profile. Each of these pages has been customized by 
the service administrator to show a selected subset of all IPS available products relevant to that user community.

There is also available a personal page where the user can select his own products of interest and display them by using widgets.

Here the user can freely customize his own personal page adding one of the available web components (e.g. image viewers, plots, maps, gauges, tables, etc.) to monitor specific performance figures of his own interest. As an example, the user can add a viewer to monitor the trend of a performance figure of one of the IPS GNSS stations close to a desired location or monitor the behaviour of ionospheric TEC focusing on a specific location or an area of his interest. Currently IPS is able to generate and make available to the users more than 160 different performance products related to the ionosphere status and its effects on GNSS.

Products are then refreshed in real-time, thereby allowing regular checks without having to reload the computation. In this sense, the web page is conceived as an operator's console.

Moreover, it is even possible to setup a watchdog alarm for one of the monitored nowcasted or forecast physical quantities to warn the user when such quantity gets outside a specified interval; the IPS is capable to timely send alarm notifications to the user by e-mail.

\section{PRODUCT VALIDATION AND MAIN RESULTS}

The objective of the validation task is to demonstrate that the products generated by the IPS prototype are issued with a sufficient confidence level; in order to understand the selected validation strategy, it is important to explain first the constraints imposed by the modelling and the engineering solutions behind the IPS products generation. The main stringent requirement for the development of the IPS service is to ensure a good level of reliability for a platform based on real-time processing. This implies that all the implemented algorithms shall avoid long periods of processing for a good user experience and for a timely provision of warnings. In the IPS system, estimation, interpolation and learning techniques were applied to speed up the processing and reach the required reliability. Some constraints used to implement the algorithms are:

a. Training: the definition and use of specific set of input/output relations to "teach" the algorithm (i.e. neural networks);

b. Fitting: definition of fitting function (linear or nonlinear) on the basis of data collection and specific models; the data collection generally covers a long temporal interval in the past and several physical events, so that the models can catch all the possible behaviours of the phenomenon to be estimated. The derived functions, fed with specific input data, are used to compute the nowcasted and forecast estimation;

c. Input data: the observation input data used for model derivation and products generation are gathered by specific sensors or scientific payloads, so the availability of historical data from these sources is limited.

The above general overview of the design drivers of the algorithms suggests that the validation can be a very difficult task. In order to overcome the difficulties, different strategies were put in place. During the research activity, each algorithm was pre-validated by using representative input data to check its performance and the reliability of the developed models. In a second stage, the validation has been carried out through two different strategies:

- The first was based on the so called "retro-validation"; the IPS platform provides in near real time a measure of the distance between the forecasting and the actual nowcasting of a specific product; in this way, the user can understand how good the prediction is. Moreover, the historical sequence of retro-validation products can be used to update day by day a statistical characterization of the behaviour of the service;

- The second approach was based on an offline comparison of the IPS nowcasted and forecast products against past external (i.e. coming from other services) products. This comparison with trusted references, like IGS, has represented a very important step in the quality verification of the IPS generated data.

\subsection{Solar Activity Related Products}

As stated above, the IPS service provides nowcasting (real time) and long term forecasting of flares, CME and SEP based on data from observations of the Sun. A statistical qualification of all the solar forecast products was realized.

Flares: IPS generates a retro-validation product that measures, for each flare forecast product, its consistency through a comparison with the corresponding nowcasting. The performance of the flare forecasting algorithm is evaluated by using the so-called Confusion Matrix (CM) [2]. In particular, the comparison between the 24-hour Mand X-class flare forecasts and the observed flare events are interpreted via a $2 \times 2$ matrix, where the entry values are expressed in terms of normalized probability.

In practice, the validation process acquires GOES-X data from the last month and compares the fluxes with the last month forecast of $\mathrm{M}$ - and X-class flare probability, and then taking into account the probabilistic nature of the forecast, it computes the $\mathrm{CM}$. The $\mathrm{CM}[0,0]$ entry will contain the percentage of correct forecast of flare occurrence (True Positive, TP), CM[0,1] the percentage of incorrect forecast of no-flare occurrence (False Positive, $\mathrm{FP}), \mathrm{CM}[1,0]$ the incorrect forecast of flare occurrence (False Negative, FN), CM[1,1] the correct forecast of noflare occurrence (True Negative, TN). Table 1 shows the $\mathrm{CM}$ for both the $\mathrm{M}$ - and X-class flares

Essentially, the validation process has foreseen the acquisition of flare data from GOES-X database and the computation of available forecast versus flare evidence in 
next 24 hours with the associated confusion matrix showed by Table 1 .

\begin{tabular}{|c|c|c|c|}
\hline \multicolumn{2}{c|}{ M Flares } & \multicolumn{2}{c|}{ X Flares } \\
\hline $\mathrm{TP}=0.0$ & $\mathrm{FP}=0.03$ & $\mathrm{TP}=0.0$ & $\mathrm{FP}=0.002$ \\
\hline $\mathrm{FN}=0.0$ & $\mathrm{TN}=0.97$ & $\mathrm{FN}=0.0$ & $\mathrm{TN}=0.998$ \\
\hline
\end{tabular}

Table 1 - M- and X-class flare probability Confusion Matrix.

CME: the parameter of interest for the CME retrovalidation is the Time of Arrival (ToA) on Earth. The IPS algorithm computes the ToA for each Earth-boud detected $\mathrm{CME}$, i.e. the time at which the particles and the magnetic field of the CME will interact with the terrestrial magnetosphere. The SEP monitoring product is used to validate whether and when the CME has hit Earth. The retro-validation is evaluated by computing the histogram of the residuals expressed as $\Delta t=t_{a p}-t_{a o}$ where $t_{a p}$ is the predicted ToA and $t_{a o}$ is the observed ToA for a given CME event. The mean value and the dispersion of this histogram are used to evaluate the performance of the prediction. The histogram of the differences between observed and forecast ToA for the catalogue of 21 CMEs published in [3] is shown in Figure 3.

The blue histogram indicates the distribution of the differences between forecast and actual arrival time (i.e. the distribution of the residual). The red line is the Gaussian fit to this histogram. The green line is the expected distribution of the differences, i.e. of the model used for the prediction. It represents the theoretical asymptotic (for an infinite number of events) histogram, which is expected from the model, given the intrinsic dispersion in the model, i.e.: a Gaussian function centred in zero.

The congruence between the red and green curves is evident and shows a small bias towards shorter ToA forecasts. The dispersion $(60 \%$ of the cases are predicted within +/- 10 hours of the actual ToA) is compatible with the model standard deviation. Therefore the forecasting can be considered satisfactory. Note: usually, a CME reaches Earth in around three to four days. For a more detailed presentation of the results, the reader can refer to [4].

SEP: The same approach as for the CME ToA was applied for the SEP flux forecasts. In this case the parameter of interest is the peak of the flux of energetic $(>10 \mathrm{MeV})$ protons of solar origin. As before, the SEP monitoring product is used to record the maximum values of $10 \mathrm{MeV}$ particle fluxes and the differences between the logarithm of the forecast peak flux and measured peak flux is computed, day by day for the last year. Again, the histogram of these differences is computed and compared to the intrinsic dispersion of the forecast model. An example of the graphical output of this product is provided in Figure 4 As in the previous figure, the red curve is a Gaussian fit to the histogram and the green curve is the expected dispersion of the model. In this case, we report the results of the retro-validation for the last year. It is evident that the forecast is performing better than expected (the histogram is centered in zero and its dispersion is much smaller than that of the green curve). However, it is worth to remember that the Sun is at present in a low activity period and, consequently, the SEP flux has been very moderate. We therefore lack enough high-flux SEP events to satisfactorily evaluate the forecast performance.

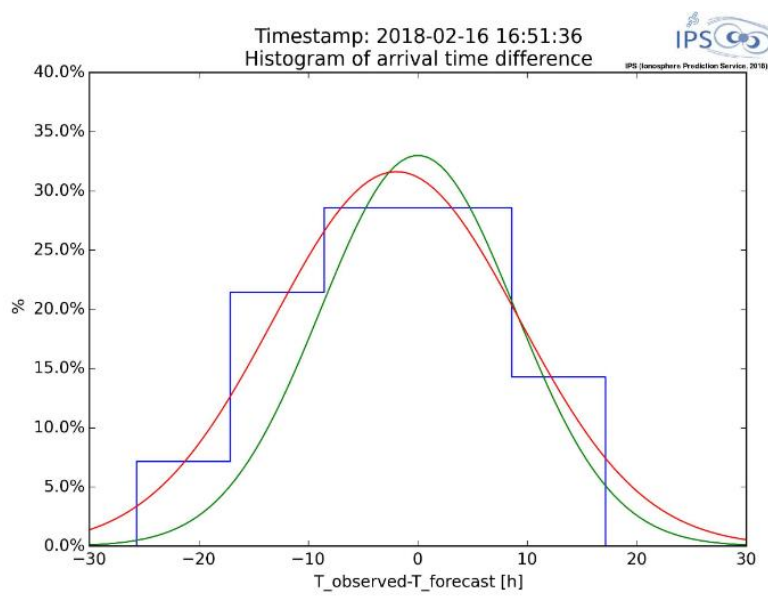

Figure 3 - CME ToA Residuals Histogram

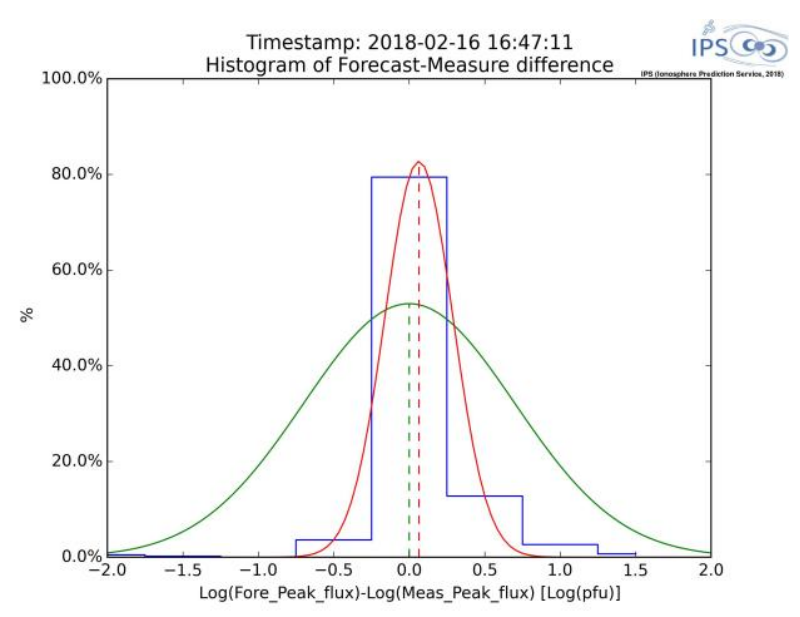

Figure 4 - SEP Peak Flux Residuals Histogram

\subsection{TEC and Scintillation Related Products}

The validation strategy here reported for the ionosphere related products, considers TEC global and regional nowcasting and the TEC global and regional forecasting (30 minutes and 24 hours). This gives 6 different TEC products ( 2 different geographical extents and 3 different prediction windows). Due to the different nature of the products a validation strategy has been chosen case by case.

The TEC global nowcasting has been compared to IGS rapid products. IGS rapid products are the product released by IGS with the smallest latency ( $<24$ hours), so they are best candidates for comparing to the IPS TEC products. 


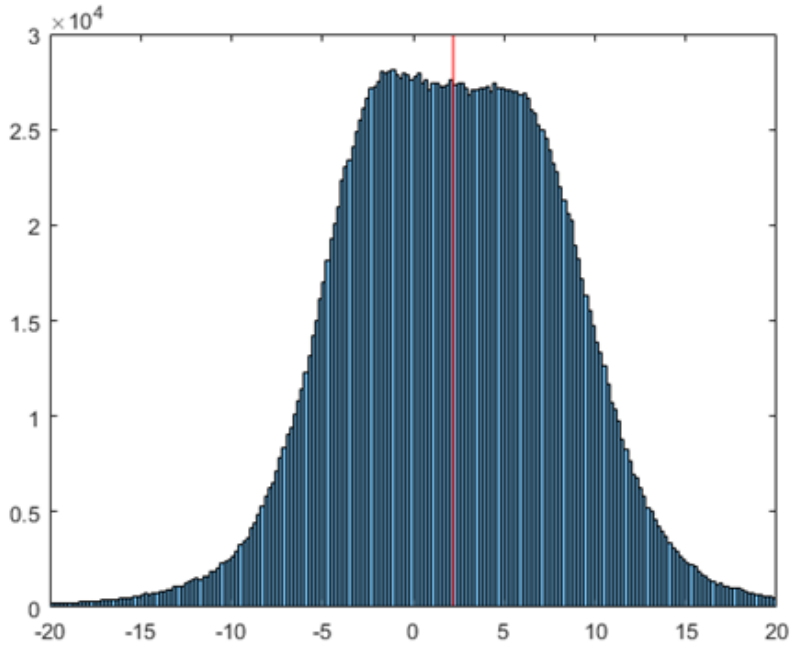

Figure 5 - Residuals Histogram of Nowcasted IPS Global TEC vs Rapid IGS

A mean of 2.19 TECu and a standard deviation of 6.15 TECu are achieved. Note: the difference between the IPS and the IGS products is in the order of the accuracy of the IGS rapid product (that is declared to be between 2 and 9 $\mathrm{TECu})$.

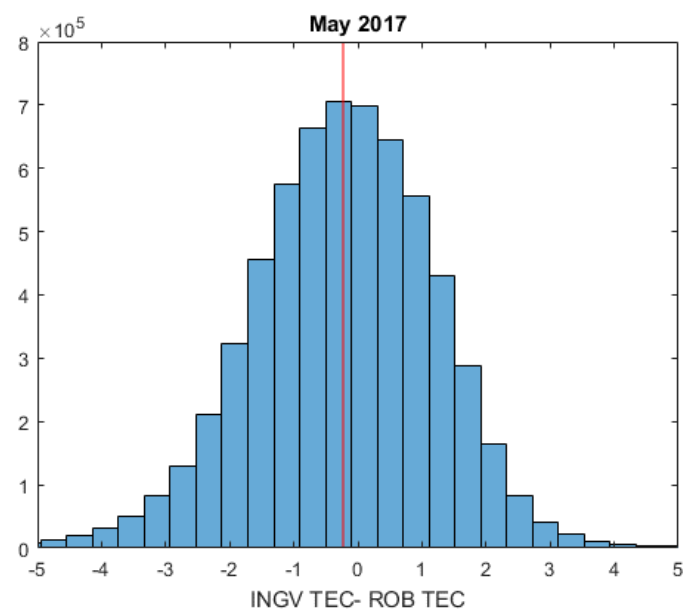

Figure 6 - Residuals Histogram of Nowcasted IPS European TEC vs Royal Observatory from Belgium

In the case of the TEC nowcasting product computed over Europe, the validation has been carried out using products from the Royal Observatory from Belgium (ROB). The analyzed period covers 2017 and it has been also extended from April 2017 to April 2018. As an example of the results, the histogram of Figure 6 represents the statistics of the differences between the two data sets for May 2017. A mean of $-0.23 \mathrm{TECu}$ and a standard deviation of 1.46 $\mathrm{TECu}$ are obtained, which is a good fit of the prediction to the reality.

Once validated, the nowcasted products were then used to check the reliability of the short term forecasting of the TEC (over Europe or global area). The short term forecasting is compared to the actual nowcasting and the difference is provided. As a first result, the histogram of the residuals is shown in the Figure 7.

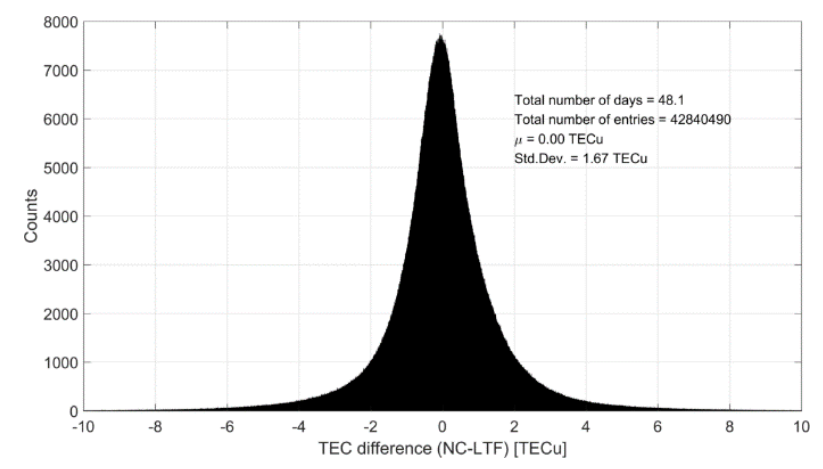

Figure 7 - Residuals Histogram of Short Term Forecast IPS Global TEC vs Corresponding IPS Nowcasting

Because of the quiet solar activity during the validation phase and the absence of significant ionospheric anomalies, we also processed historical data in order to check the behaviour of the algorithms in presence of events of different magnitude

For each event, the validation approach was as follows:

a. Nowcasting (NC) and short term forecasting (STF) of TEC over Europe validated by using as external product the ROB European NC TEC maps (time resolution: 15 minutes) from Royal Observatory of Belgium (ROB) available at ftp://gnss.oma.be;

b. NC and STF of TEC at global level validated by using as external product the rapid global TEC maps (time resolution: 15 minutes, latency: less than 24 hours) from Universitat Politècnica de Catalunya (UPC) available at ftp://cddis.gsfc.nasa.gov;

c. Long-Term Forecasting (LTF) of TEC at global level validated by using as external product the final global TEC maps (time resolution: 2 hours, latency: approximately 11 days) from Universitat Politècnica de Catalunya (UPC) available at ftp://cddis.gsfc.nasa.gov.

The details about validation of the TEC and scintillation related products are reported in [5] but, summarizing, the validation exercise indicates a very high accuracy of the IPS TEC products. The standard deviation of the residuals is below 1.9 TECu for the European products, and below 5 $\mathrm{TECu}$ for the global products. This indicates a good precision of the IPS TEC products during high geomagnetic activity.

\subsection{GNSS Receiver Performance Related Products}

The GNSS receiver related products are generated by the RPF 3 module and provide meaningful information to end users, through tools for the estimation of:

- GNSS receiver tracking errors;

- Probability of loss of lock;

- User positioning errors.

All the estimated values represent the effect on the final user receiver performance due to the level of scintillation 
activity. The impacts of scintillation were modelled through three sets of parameters and associated fitting functions corresponding to high, medium and low latitude. Therefore the validation must take into account this distinction. Regional nowcasting products from the available GNSS reference stations were taken into account for the three different latitudinal sectors. The models were validated under two conditions: days when there was scintillation activity and days when there wasn't scintillation.

The validation process was carried out in two stages and was valid for both nowcasting and forecasting products.

For the first stage during the selected time interval the raw measurements were extracted from the stations and fed into the models that were used to compute the different parameters. The trends of the estimated parameters through the developed models were compared to the trends of the real parameters measured from the same stations. Therefore an analysis on the correlation between the two trends is possible and the correlation coefficient represents the level of correctness of the estimation and prediction, i.e. high correlation $(>0.5)$ means that the model is able to capture the real trends.

The second stage repeated the same process as in the first stage, but in this case, it considered the use of the RPF 2 outputs as input to the models of the RPF 3 instead of using the real measurements coming from the stations.

The RPF 3 regional products were validated using data recorded by ISMRs operational at the European high and middle latitudes. The RPF 3 global products have been validated using data recorded by ISMRs operational at the high and middle latitude stations over Europe and North America and at the low latitude stations over Brazil.

The goodness of fit of the developed model algorithms for tracking error and user positioning error products was evaluated by using the Root Mean Square Error (RMSE) of the residuals. The RMSE is a measure of how accurately the model estimates the observations themselves and a low value of the RMSE indicates the closeness between the observations and their model estimations. As the loss of lock products measure a probability, the goodness of the developed models was evaluated using the correlation coefficient, R. R is a measure of how well the model estimates the variability in the observations and a higher value for $\mathrm{R}$ indicates that the model represents the trend of the observations well. The details on the validation results are discussed in [5].

The validation results for tracking error products indicated that the RMSE values were all below $0.1 \mathrm{~mm}$, which is one order of magnitude smaller than the precision of the GPS L1 carrier phase measurements, of about $2 \mathrm{~mm}$. This indicates that the values of the residuals are well below the L1 tracking noise level, thus suggesting a good performance of the developed models.

The validation results for the user positioning error products indicated that the RMSE values were all below 10 $\mathrm{cm}$, which is within the expected positioning accuracy from an epoch-wise Precise Point Positioning (PPP) solution. The validation results for the probability of loss of lock products indicated that the $\mathrm{R}$ values were all close to 1, suggesting a good performance of the developed models, as they are able to properly recover the variability observed in the probability of loss of lock.

\subsection{GNSS System Performance Related Products}

This paragraph presents the validation approach that has been used for the products generated by the RPF4 regarding the final performances of the GNSS system as seen by a user belonging to a specific community in terms of accuracy of positioning error, integrity and availability. We remind that RPF4 generates the following predictions:

- Short-term forecasting of the standalone positioning error over a global area; the error is computed in correspondence of the regular nodes of a virtual grid of GNSS receivers;

- Short-term forecasting of the ABAS availability over a global area according to the FDE-RAIM algorithm (Fault Detection and Exclusion Receiver Autonomous Integrity Monitoring); as before the performance is computed for an artificial network of GNSS receivers;

- Short-term forecasting of the ABAS integrity in term of prediction of FDE-RAIM horizontal protection level; also in this case the map is computed interpolating over a grid of virtual GNSS receivers.

According to what said, the validation has been carried out by comparing the forecast results with nowcast information determined for the same epochs and computed by using the observation measurements and the navigation data acquired by a set of real GNSS receivers.

Since we don't have the positioning output as processed by the hardware receivers, the same nowcasting products are the outcomes of internal developed algorithms. This means that also the nowcasting processing has been validated.

The selected strategy to validate nowcasting analysis is to statistical compare the outcomes of the RPF4 positioning algorithm with the output results given by the Telespazio Galileo Service Operation (GSOp) User Terminal (UT). The Telespazio UT is a multi-constellation software tool able to compute the position of a specific GNSS terminal given its observation and navigation data. It has been validated in the context of the GSOp program and is currently running in the Key Performance Indicators (KPI) tool at Fucino Galileo Control Center for the monitoring of the Galileo navigation performances. The comparison will consider the following statistical parameters for both solutions:

- Total position error $95 \%$ accuracy (calculated as $95^{\text {th }}$ percentile of error time series);

- Mean total position error;

- Maximum total position error.

The analysis has been performed processing the data of the reference Ionospheric Event from 07 to 11 September 2017 for the following three EUREF Stations: Kiruna (SW), Brest (FR) and Canary Island (ES). 
A summary of the obtained results is reported in Table 3. Overall, we can observe a substantial impact on the Kiruna station and Canary Island positioning performance.

The performance results were compared using the above mentioned algorithms. Different algorithm configuration options may produce different output results when observing the complete error time-series, typically in case of extreme events, like outliers, peaks, etc.:

\section{- Tropospheric models (US-STD / UNB-3);}

- Outlier detection algorithms (when enabled);

- Solution weighting schemes.

These issues were taken into account to justify discrepancies between the two algorithms.

Nevertheless, compared results between the two independent KPI analyses shows a substantial correspondence.

Forecast validation methodology consists of comparing performance results achieved at station level, that is the output of real GNSS receiver observations processing, with position error forecast maps, available for a specific region, at the corresponding station location coordinates and at the same time of data validity.

Position error forecast at a given sample point is considered reliable if corresponding regions of estimation uncertainty partially or fully overlap.

Significant discrepancies may occur at critical sites where environmental and siting conditions cannot be taken into account by the forecast algorithms (because partially based on models) and due to simulation grid resolution constraints to satisfy computational requirements and achieve results in a reasonable time.

Due to such limitations, this approach can result too conservative to demonstrate the validity of the forecast algorithm.

First results revealed discrepancies between nowcast (output of processing at station level) and forecast (output of volume simulator) in the order of 1-2 meters for some network stations presumably depending upon:

- Error in forecast ionospheric maps;

- Precise orbit and clocks (6h predicted interval of the IGS ultra-rapid SP3 products);

- Under-modelled user error sources (dominant effect): siting and local environmental characteristics (e.g. signal obstructions due to the presence of reliefs, effect of multipath, etc.);

- Receiver noise and related intrinsic technical specifications, model, type and age.
Forecast approach limits are clear but the methodology remains valid: this method can forecast performance figures very closely to the real future ones.

\section{CONCLUSIONS}

The prototype started the operations on July 2018, for a 6 month period. The several user communities were invited to test the platform and the relevance of its functions and products for specific use cases.

During the same phase, user feedback on the service concept (suggestions for new features, interface improvements, but also to report anomalies, etc) will be collected, notably through dedicated workshops. After this important phase of direct feedback from the users, the IPS prototype will gain sufficient inputs to evolve in a more mature service.

IPS is a service devoted to the whole GNSS community and for this reason the IPS service is planned in the future to be provided by the EU GNSS Service Centre (GSC) located in Madrid, Spain.

The role of the GSC is to inform users about the status of the service pushing notification to the GNSS users/communities about performance, and IPS will have to meet in its evolution the GSC specific needs and adapt accordingly.

\section{REFERENCES}

[1] E. Guyader, F. Rodriguez, R. Ronchini, S. Di Rollo, Ionosphere Prediction Service for GNSS Users, ICAO Working Paper, Navigation Systems Panel (NSP) Joint Working Groups Meeting, Montreal, June 2017.

[2] T. Fawcett, An Introduction to ROC Analysis, Pattern Recognition Letters. 27, 861, 2006

[3] T. Shi at al., Predicting the Arrival Time of Coronal Mass Ejections with the Graduated Cylindrical Shell and Drag Force Model, The Astrophisical Journal, 2015.

[4] G. Napoletano, R. Forte, D. Del Moro, E. Pietropaolo, L. Giovannelli, F. Berrilli, A Probabilistic Approach to the Drag-Based Model, Journal of Space Weather Space Clim., Volume 8, 2018.

[5] Vadakke Veettil S. et al The Ionosphere Prediction Service Project: Translating ionospheric information into GNSS user devoted metrics submitted to the Journal of Space Weather Space Climate. 


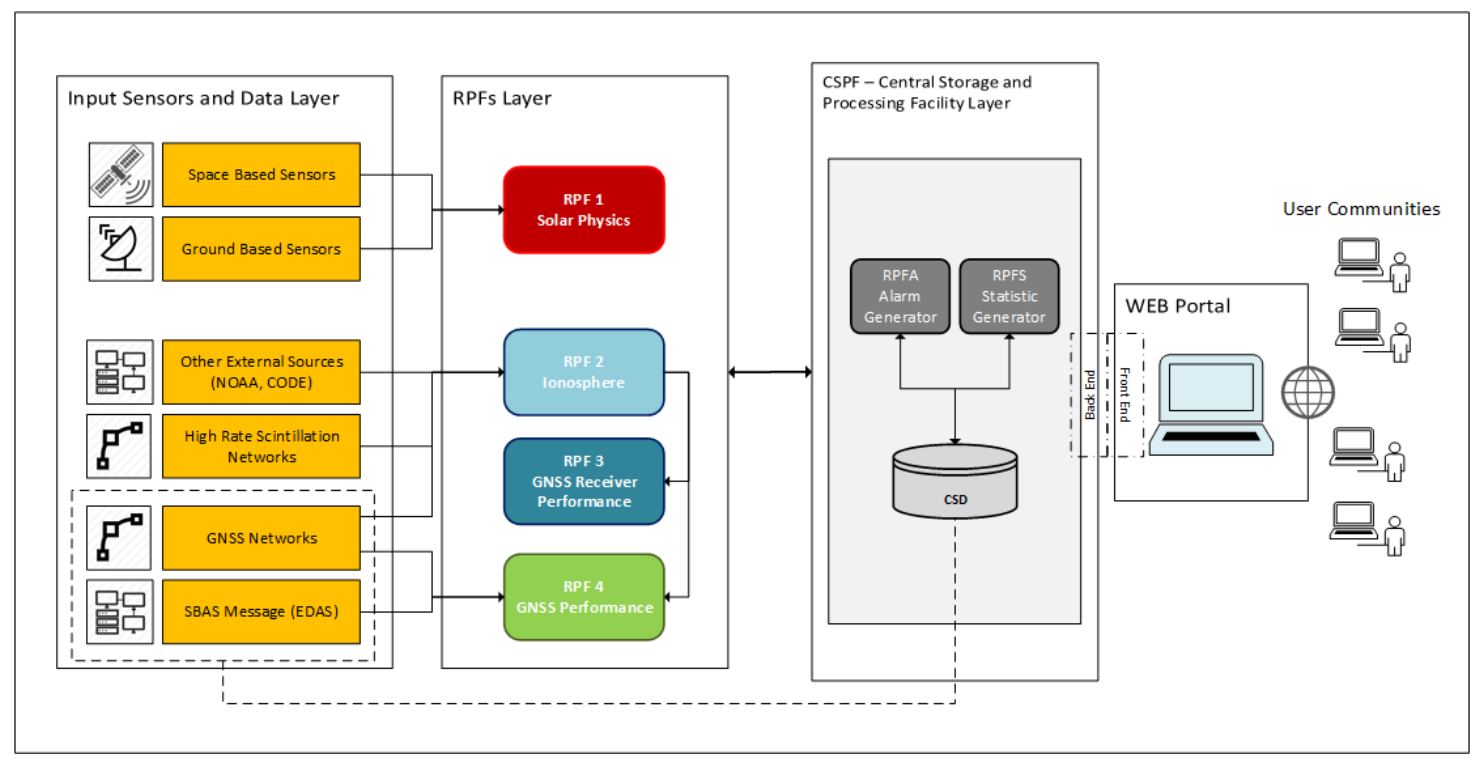

Figure 8 - IPS Logical Architecture

\section{APPENDIX B}

\begin{tabular}{|c|c|c|c|c|c|}
\hline Product Generator & Product Category & NC Refresh Rate & $\begin{array}{l}\text { ST Forecasting } \\
\text { Horizon / Rate }\end{array}$ & $\begin{array}{l}\text { ST Forecasting } \\
\text { Horizon / Rate }\end{array}$ & Extent \\
\hline \multirow{3}{*}{ RPF1 } & Flare & $6 \mathrm{~h}$ & N/A & $24 h / 1 h-6 h$ & - \\
\hline & $\mathrm{CME}$ & $1 \mathrm{~h}$ & N/A & $24 \mathrm{~h} / 6 \mathrm{~h}$ & - \\
\hline & SEP & $15 \mathrm{~min}$ & N/A & $24 \mathrm{~h}-1 \mathrm{~h}$ & - \\
\hline \multirow{3}{*}{ RPF2 } & TEC & $15 \mathrm{~min}$ & $30 \mathrm{~min} / 15 \mathrm{~min}$ & $24 \mathrm{~h} / 2 \mathrm{~h}$ & Global / Europe / Italy \\
\hline & Scintillation & $15 \mathrm{~min}$ & $30 \mathrm{~min} / 15 \mathrm{~min}$ & - & Europe \\
\hline & PSI & $15 \mathrm{~min}$ & $30 \mathrm{~min} / 15 \mathrm{~min}$ & $24 \mathrm{~h} / 3 \mathrm{~h}$ & Global \\
\hline \multirow{4}{*}{ RPF3 } & Tracking Error & $15 \mathrm{~min}$ & - & $24 \mathrm{~h} / 3 \mathrm{~h}$ & Global / Europe \\
\hline & Positioning Error & $15 \mathrm{~min}$ & - & $24 \mathrm{~h} / 3 \mathrm{~h}$ & Global / Europe \\
\hline & Loss of Lock Prob & $15 \mathrm{~min}$ & - & $24 \mathrm{~h} / 3 \mathrm{~h}$ & Global / Europe \\
\hline & TID & $15 \mathrm{~min}$ & - & - & Italy \\
\hline \multirow{4}{*}{ RPF4 } & $\begin{array}{l}\text { ABAS Position Error and } \\
\text { RAIM Integrity Availability } \\
\text { Analysis: Summary Table with } \\
\text { all Monitored Sites }\end{array}$ & $1 \mathrm{~h}$ & - & - & On Site (Network) \\
\hline & $\begin{array}{l}\text { ABAS Position Error and } \\
\text { RAIM Integrity Availability } \\
\text { Analysis: Detailed Performance } \\
\text { Report and Time-Series for } \\
\text { Worst-Case Site }\end{array}$ & $5 \mathrm{~min}$ & - & - & On Site \\
\hline & $\begin{array}{l}\text { ABAS Position Error and } \\
\text { RAIM Integrity Availability } \\
\text { Analysis }\end{array}$ & - & $\begin{array}{l}30-45 \min / 15 \\
\min \end{array}$ & - & Global / Europe \\
\hline & $\begin{array}{l}\text { SBAS LPV Availability and } \\
\text { Continuity Analysis }\end{array}$ & $5 \mathrm{~min}$ & - & - & Europe \\
\hline
\end{tabular}

Table 2 - IPS Products Summary

International Technical Symposium on Navigation and Timing (ITSNT) 2018

13-16 Nov 2018

ENAC, Toulouse, France 
APPENDIX C

\begin{tabular}{|l|c|c|c|c|c|c|}
\hline \multicolumn{1}{|c|}{ EUREF Station } & \multicolumn{2}{|c|}{ Position Error 95\% accuracy } & \multicolumn{2}{c|}{ Mean Position Error } & \multicolumn{2}{c|}{ Maximum Position Error } \\
\hline & Algorithm 1 & Algorithm 2 & Algorithm 1 & Algorithm 2 & Algorithm 1 & Algorithm 2 \\
\hline $\begin{array}{l}\text { KIR0 } \\
\text { (Kiruna, SW) }\end{array}$ & $6.59 \mathrm{~m}$ & $6.80 \mathrm{~m}$ & $3.35 \mathrm{~m}$ & $3.58 \mathrm{~m}$ & $10.82 \mathrm{~m}$ & $10.44 \mathrm{~m}$ \\
\hline $\begin{array}{l}\text { BRST (*) } \\
\text { (Brest, FR) }\end{array}$ & $4.68 \mathrm{~m}$ & $4.40 \mathrm{~m}$ & $2.41 \mathrm{~m}$ & $2.34 \mathrm{~m}$ & $8.90 \mathrm{~m}$ & $9.01 \mathrm{~m}$ \\
\hline $\begin{array}{l}\text { LPAL } \\
\text { (Canary Islands, ES) }\end{array}$ & $8.07 \mathrm{~m}$ & $8.80 \mathrm{~m}$ & $4.03 \mathrm{~m}$ & $5.27 \mathrm{~m}$ & $12.11 \mathrm{~m}$ & $13.81 \mathrm{~m}$ \\
\hline
\end{tabular}

Table 3 - Validation Results of Nowcasted Positioning Error 